Rejected words: Palliative, Holistic, Specialist, Rehabilitative, Care in the last days

Conclusion Phyllis Tuckwell Hospice Care provides supportive and end of life care for patients and families living with a terminal illness.

\section{P-215 PATIENTS' UNDERSTANDING OF TERMINOLOGY USED IN PALLIATIVE CARE SERVICES}

${ }^{1}$ Abi Ponnampalam, ${ }^{2}$ Nicola Holtom, ${ }^{2}$ Sanda Hlaing. ' Garden House Hospice, Letchworth Garden City, UK; ${ }^{2}$ Norfolk and Norwich University Hospital, Norwich, UK

\subsection{6/bmjspcare-2016-001245.236}

Background Good communication stipulates that we avoid using jargon. However patients' understanding of terms such as 'specialist palliative care nurse' and 'Macmillan nurse' seems variable. We are often asked by patients who are already known to a palliative care specialist nurse, whether they could also see a Macmillan nurse.

In addition, as palliative care evolves and the role of hospices has changed to include complex symptom control, the subsequent name change to 'Specialist Palliative Care Unit' seems logical. However patients' understanding of this seems variable.

Aim To establish the understanding patients have of the terms commonly used in palliative care and ascertain whether the terms we take for granted cause any confusion.

Methods Qualitative semi structured interviews with 20 participants attending palliative care outpatient clinics. Participants had a range of life-limiting conditions and prognoses. Patients new to the service and those already known were sampled to identify any difference in their understanding. Interviews were recorded and transcripts were analysed using the principles of thematic analysis.

Results Only four patients were able to identify that the roles of specialist palliative care nurses (SPCN) and Macmillan Nurses were similar. Four patients felt that the SPCN was the same as the cancer specialist nurse. Five patients specifically mentioned the Macmillan nurse being available to provide hands on basic nursing and overnight care.

All participants identified end-of-life care with the term hospice. More than half did not know what a Specialist Palliative Care Unit entailed. Almost a third thought it was the same as the acute oncology ward or chemo/radiotherapy unit. Only three patients expressed their understanding of the two providing a similar role.

Conclusion There seems to be a real discrepancy between what is meant by these terms used commonly in practice and what is actually understood by patients. This raises serious issues which would benefit from further study

\section{P-216 INTERVAL}

Steven Eastwood, Peter Gilliam. Earl Mountbatten Hospice, Newport, UK

\subsection{6/bmjspcare-2016-001245.237}

Introduction Non-fiction filming involving death and dying has taboo status in terms of what western society can and cannot sanction. Made over the course of 12 months through close engagement with individuals experiencing and witnessing death and dying, our film consists of long sequences showing the people, environments and activities in and around the hospice, exploring what happens at the end of a person's life.

The project Upon filming it became clear that the hospice acts as the beating heart at the centre of the island, with its community team operating as an artery system, extending out to every part of the land. In many ways this is a palliative island, a place where dying is visible. With this notion of an island as ecology, one that is familiar with end of life, the filmmaker began to notice complex and coexistent movements and practices associated with dying. Filming with people in family homes and on the ward during the last weeks of life unfolded to include the very intimate moment of the instant of death. Filming also extended out into the landscape, taking in chemotherapy, bereavement seminars, pathology lab activity, but also ferry crossings, druid death ceremonies, and palliative care given to ageing big cats at the zoo.

The project attempts to engender a space where the phenomenon and phenomena of dying and death can be given an image. This reflects changing attitudes in palliative care and society around the visibility of death and dying. Navigating this difficult ethical territory involved adopting a way of seeing, and being with, the terminally ill person that has some confederacy with the practices of the palliative care professional. What emerges is a slow cinema description of the temporality of dying, an image of care and attentiveness and the very natural process of death.

\section{P-217 COMMUNICATION PASSPORT PROJECT}

${ }^{1}$ Ruth Butcher, ${ }^{1}$ Leanna Tuffin, ${ }^{1}$ Natasha Harding, ${ }^{1}$ Liz Lewington, ${ }^{2}$ Sophie Dziwinski. ${ }^{1} J e s s i e$ May, Bristol, Great Britain; ${ }^{2}$ Roald Dahl; ${ }^{3}$ Burdett Trust

\subsection{6/bmjspcare-2016-001245.238}

Jessie May is a charity-run organisation who provides specialist care and support through a team of children's nurses and nursery nurses in partnership with families and other agencies to deliver respite and palliative care service in the comfort of the families own home to children and young people with life limiting conditions.

The Departments of Health and Education (2015) define children with complex and continuing health care needs as individuals with prolonged chronic conditions which have an adverse effect upon a child's development and welfare. Many of the children with long term life-limiting illness or disabilities may have impaired communication (Teare, 2009).

However Hewitt-Taylor (2008) suggests that communication difficulties may not arise because of the child's inability to understand, but occurs through inability to make themselves understood by others. Children with complex needs communicate about the same things as the other children, for example their feelings, needs, likes and dislikes (Mencap, 2010). The Convention on the Rights of a Child (2014), specifically states that each and every child has a right to be listened to. Jessie May has recognised the possible barriers for some of the children that we care for being heard and listened to due to communication barriers. This problem is something that communication passports address.

A literature review identified three pieces of research, Millar (2007), Sanderson (2003) and Bell, 2012), all have similar findings that suggest there are significant benefits to using communication passports, and we aim to conduct a study on communication passports to ensure that we produce a passport that is relevant to the service that we provide. 